\title{
Sistemas de Información Geográfica (SIG) y Metodologías de Evaluación Multicriterio (EMC) en la búsqueda de escenarios alternativos para el mejoramiento socio-espacial de las áreas urbanas populares de la Ciudad de Comayagua
}

José Luis Palma Herrera

\section{Resumen}

La investigación brindo una metodología en base a SIG y EMC como herramientas de apoyo para que el gobierno local mejore la accesibilidad espacial de la población menos favorecida de la ciudad de Comayagua a servicios públicos.

Fases de la aplicación: 1) Obtención de mapas vector: se generan los mapas de todas las centralidades: i) puntuales: centros de salud y centros educativos; ii) lineales: rutas de transporte público. 2) Obtención de criterios: Los vectores se transforman en ráster, de esta manera, se puede trabajar con sus pixeles como unidad de medida y evaluación. La evaluación Multicriterio se realiza con el método WLC. 3) Obtención de criterios: cada centralidad o vector es una "capa temática". Luego se define para cada una las distancias a la centralidad. Se definirán franjas de similar amplitud (metros) alrededor de la centralidad. Los valores de los pixeles para cada franja serían 5, 4, 3, 2 y 1 los pixeles más lejanos. 3) Combinación lineal ponderada (WLC): Al tener todos estos mapas de "nivel de accesibilidad" a cada centralidad o mapa, se superponen o suman con la calculadora. El valor mayor será el de mayor accesibilidad y el de menor valor el de menos accesibilidad. Esta resolución simple luego puede obtener variantes con el método de ponderación por WLC donde previo a la suma de valores se multiplican por un valor ponderado previamente. Pero se aplica posteriormente de haber obtenido los primeros resultados. Este es el MAPA DE ACCESIBILIDAD, es el resultado u objetivo 1. 4) Evaluación del modelo: realizamos una evaluación con criterios de accesibilidad para el cumplimiento de los objetivos del estudio. Si el modelo cumple dichos criterios el objetivo es 
alcanzado. En caso contrario, se genera otro modelo territorial para luego aplicar el procedimiento nuevamente hasta llegar al modelo óptimo.

Palabras clave: Evaluación Multicriterio; Ordenamiento Territorial; Barrios Populares; Accesibilidad; Criterios de decisión.

José Luis Palma Herrera (herrer@hotmail.com). Universidad Nacional Autónoma de Honduras (UNAH), Facultad de Ciencias Espaciales (FACES), Maestría en Ordenamiento y Gestión del Territorio. Boulevard Suyapa, Ciudad Universitaria, F.M., Tegucigalpa, M.D.C., Honduras, Centroamérica. 


\section{INTRODUCCIÓN}

Las ciudades latinoamericanas han crecido hasta sobrepasar por mucho su capacidad para brindar a los ciudadanos un entorno productivo que ayude a la gestión pública en servicios que cubran necesidades públicas. Se ha llegado a una situación en la cual se hace evidente la carencia de adecuados servicios, un ineficiente manejo de la educación, salud y vías de comunicación, lo cual está causando un marcado descenso en el nivel de calidad de vida de la población en áreas metropolitanas y comunidades cercanas. La ya precaria infraestructura se está socavando debido a la presión proveniente de la creciente migración y la proliferación de barrios populares 0 barriadas marginales en las ciudades.

En este sentido, resulta evidente que, uno de los principales problemas de la gestión gubernamental está dado por el uso de las herramientas de gestión y toma de decisiones. En los últimos años, cada vez es más común utilizar Sistemas de Información Geográfica (SIG) para la toma de decisiones, los cuales pueden brindar información socio-espacial relevante en múltiples dimensiones. A través de la modelización digital es posible apoyar una actividad de racionalidad científica en la planificación territorial.

En conclusión, abordando las comunidades de una ciudad, como una red, las autoridades que formulan políticas de desarrollo pueden lograr un mejor equilibrio espacial en la política y gestión de desarrollo económico. Esto se logrará realizando acciones correctas, y para esto se necesitan las herramientas idóneas con fines a la toma de decisiones con base científica. En una política tal, se reconocerá que la satisfacción de las necesidades básicas y el bienestar de la población de las áreas metropolitanas dependen del fortalecimiento de la base económica y la infraestructura social de los asentamientos más necesitados. Y para la toma de decisiones y gestión de tierras, se consideran de gran utilidad las herramientas de administración de información territorial.

\section{AREA DE ESTUDIO}

La investigación de la presente tesis tiene como área de estudio la ciudad de Comayagua cabecera del Municipio y del Departamento homónimo. Ubicado en la zona central de la Republica de Honduras en Centroamérica. 
Figura 1. Mapa No.1 Ubicación geográfica de la Ciudad de Comayagua Ciudad de Comayagua

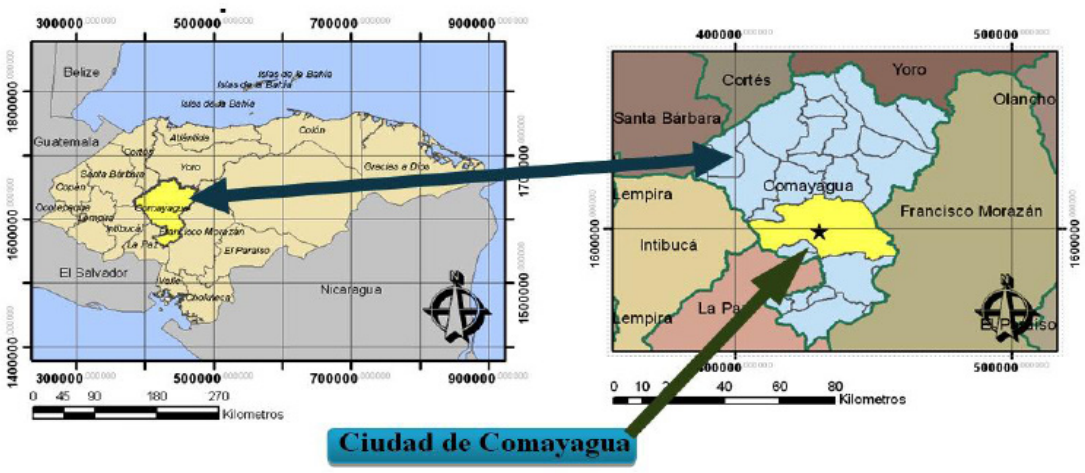

Fuente: elaboración propia.

La ciudad de Comayagua está localizada a unos 594 metros sobre el nivel del mar. Al sur de Comayagua se encuentra Tegucigalpa (80 kilómetros) y al norte la ciudad de San Pedro Sula (165 kilómetros). La ciudad tiene una población de 50,600 habitantes y la superficie municipal tiene 5,124 kilómetros cuadrados.

La ciudad de Comayagua está formada por barrios y colonias, como todas las ciudades de Honduras. Y de acuerdo al relevamiento de los servicios básicos de la población y uso del espacio físico del año 2,000 (ver anexo No.1), realizado por la Unidad de Investigación y Estadística Social de la Municipalidad de Comayagua (UIES-COM) y con financiamiento por las naciones unidas, se identificó que los asentamientos humanos con mayor concentración poblacional son; la Colonia 21 de Abril, y los barrios; Arriba, Independencia, Abajo, Cabañas y la Sabana. Como lo muestra la siguiente tabla:

Tabla 1. Barrios y colonias con mayor concentración poblacional. Asentamientos con mayor Concentración Poblacional No. Barrio/Colonia Población \%

\begin{tabular}{|l|l|l|l|}
\hline \multicolumn{4}{|c|}{ Asentamientos con mayor Concentración Poblacional } \\
\hline No. & Barrio/Colonia & Población & $\%$ \\
\hline 1 & Barrio Arriba & 5,547 & 9.90 \\
\hline 2 & Barrio Independencia & 3,509 & 6.30 \\
\hline 3 & Barrio Abajo & 3,499 & 6.20 \\
\hline 4 & Barrio Cabañas & 3,240 & 5.80 \\
\hline 5 & Colonia 21 de Abril & 3,089 & 5.50 \\
\hline 6 & Barrio La Sabana & 2,860 & 5.10 \\
\hline
\end{tabular}

Fuente: Alcaldía de Comayagua, año 2000. 
Igualmente, este relevamiento identifico estos barrios como las zonas poblacionales con mayor concentración de unidades habitacionales ocupadas (hacinamiento de viviendas). Los resultados tabulados que demuestran esto son los siguientes:

Tabla 2. Barrios y colonias con mayor concentración de unidades habitacionales ocupadas (viviendas)

\begin{tabular}{|l|l|l|l|}
\hline \multicolumn{4}{|c|}{ Asentamientos con mayor Concentración de unidades } \\
\hline No. & Barrio/Colonia & Unidades & $\%$ \\
\hline 1 & Barrio Arriba & 1,287 & 11.0 \\
\hline 2 & Barrio Independencia & 793 & 6.80 \\
\hline 3 & Barrio Abajo & 766 & 6.50 \\
\hline 4 & Barrio Cabañas & 720 & 6.10 \\
\hline 5 & Colonia 21 de Abril & 591 & 5.00 \\
\hline 6 & Barrio La Sabana & 573 & 4.90 \\
\hline & Total & $\mathbf{4 , 7 3 0}$ & \\
\hline
\end{tabular}

Fuente: Alcaldía de Comayagua, año 2000.

De estas 4,730 unidades habitacionales, el $27.55 \%(1,303)$ tiene piso de tierra en sus interiores y el $39.20 \%$ tienen por cubierta, techos de teja. Todos los anteriores indicadores muestran que estos barrios poseen características de asentamientos populares o "barrios populares".

Debido a que estos asentamientos se han identificado como "barrios populares" podemos deducir que estas zonas residenciales contienen la población urbana con mayor necesidad de servicios para satisfacer sus necesidades básicas. Por ende, y aplicando el principio de "Justicia y Equidad Social", donde se maneja el axioma de que "los que tienen menos necesitan más", este estudio se centra en esta zonas de la ciudad; para lo cual, los mapas proporcionados por la alcaldía de Comayagua, son los insumos utilizados para generar los modelos territoriales. A continuación se muestra un mapa de estos barrios:

\footnotetext{
${ }_{1}^{1}$ Moreno Jiménez, A. 2006. En torno a los conceptos de equidad, justicia e igualdad espacial. Huellas. La Pampa. Argentina. 11:133-142
} 
Figura 2. Mapa No.2 Barrios y colonias con mayor concentración poblacional de la Ciudad de Comayagua

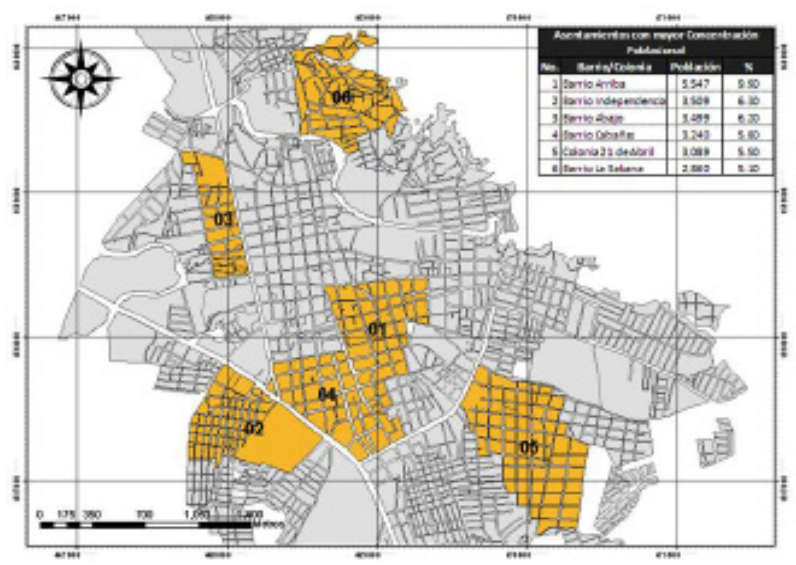

Fuente: elaboración propia.

\section{OBJETIVO DE LA INVESTIGACIÓN}

Generar un modelo territorial mediante una herramienta de ordenamiento y gestión territorial para crear escenarios que ayuden a distribuir equitativamente y de acuerdo a los más necesitados la oferta de servicios públicos y privados en la ciudad de Comayagua en base a la accesibilidad.

\section{APLICACIÓN TÉCNICA DEL EMC A CASO COMAYAGUA}

La investigación brindara elementos de decisión territorial y, con ello, convertirse en una herramienta de apoyo para que el gobierno local (organismos de planificación) implemente políticas que tiendan a mejorar la accesibilidad espacial de la población de la ciudad de Comayagua. La accesibilidad hacia diferentes "centralidades". Estas centralidades corresponden a servicios e infraestructuras urbanas. A continuación se detallan las fases de la aplicación:

\section{a. Obtención de mapas vector:}

Para la tarea de aplicación hay que tener los mapas de todas las centralidades posibles: (1) puntuales - centros de salud y centros educativos. (2) lineales - rutas de transporte público. Dichos mapas vectores, fueron proporcionados por la Unidad de Investigación y Estadística Social de la Municipalidad de Comayagua (UIES-COM). 


\section{b. Obtención de criterios:}

La evaluación Multicriterio realizada con el método WLC, trabajada en Idrisi, requiere que los vectores sean transformados en ráster. De esta manera, se puede trabajar con sus pixeles como unidad de medida y evaluación.

\section{c. Generación de factores:}

Cada centralidad o vector es una "capa temática". Luego se define para cada una las distancias a la centralidad. Ej: zonas (buffer) de diferentes distancias a los centros de salud y centros educativos. Todo en "sistema ráster" del Spatial Analyst. Se definirán franjas de similar amplitud (metros) alrededor de la centralidad, para este caso, 5 y la última una zona lejana. Los valores de los pixeles para cada franja serían 5, 4, 3, 2 y 1 los pixeles más lejanos.

\section{d. Combinación Lineal Ponderada (WLC):}

Al tener todos estos mapas de "nivel de accesibilidad" a cada centralidad o mapa, se superponen o suman con la calculadora. El valor mayor será el de mayor accesibilidad y el de menor valor el de menos accesibilidad, o puede obtenerse un "promedio" de valores, Si un pixel obtiene el valor 5 esto significa que su posición es la mejor de todas en todos los casos, 1 sería lo inverso. Esta resolución simple luego puede obtener variantes con el método de ponderación por WLC (Combinación Lineal Ponderada) donde previo a la suma de valores se multiplican por un valor ponderado previamente. Pero se aplica posteriormente de haber obtenido los primeros resultados. Este es el MAPA DE ACCESIBILIDAD DEL AREA DE ESTUDIO 1, es el resultado u objetivo 1.

\section{e. Evaluación de modelo:}

El mapa de accesibilidad y los mapas de aptitud anteriores muestran donde se localizan las zonas con mayor accesibilidad. Para determinar si el modelo es el adecuado con estos resultados, realizamos una evaluación en base a criterios de accesibilidad definidos en base al cumplimiento de los objetivos del estudio. Si el modelo cumple dichos criterios el objetivo es alcanzado y por ende el modelo es el deseado u óptimo.

En caso contrario, donde estos criterios no cumplen, se genera otro modelo territorial con la agregación o reubicación de cualquier centro (educación, salud, ruta de transporte, etc) y aplicar el procedimiento nuevamente para llegar a un segundo resultado final: MAPA DE ACCESIBILIDAD DELAREADE ESTUDIO 2. Este es el resultado 2, en el que igualmente se evaluara el cumplimiento de los criterios. 
Este proceso se continúa hasta encontrar el resultado deseado. El siguiente flujo de proceso explica gráficamente la aplicación de este caso:

Figura 3. Flujo de proceso de aplicación de Evaluación Multicriterio

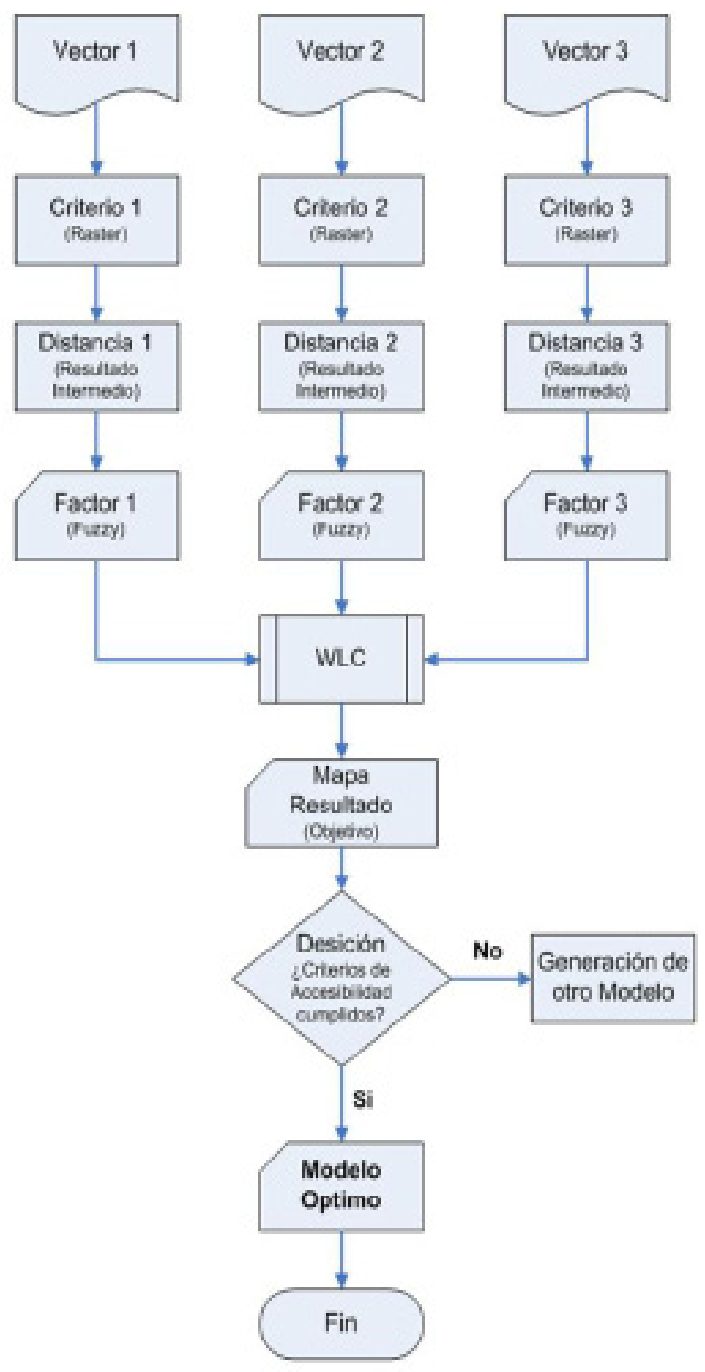


Al generar los ESCENARIOS DE MEJORAMIENTO DE ACCESIBILIDAD, se deberá seleccionar el MEJOR ESCENARIO. Para esto, utilizaremos los criterios de decisión, los cuales han sido establecidos basándose en la Justicia y Equidad espacial².

Los criterios de decisión que se utilizaran para definir el modelo territorial óptimo serán:

a) Mayor área de población beneficiada con servicios.

b) Mayor distribución de servicios.

c) Las áreas con mayor aptitud están en los barrios populares.

Con esto trataremos el problema de donde situar un servicio o actividad, escogiendo el mejor modelo territorial que logre ofrecer a la población más necesitada lo siguiente:

1. Aumentar la calidad de vida de la población que reside o usa el territorio 2. La organización más equilibrada o coherente del espacio geográfico de acuerdo a las necesidades insatisfechas.

\section{RESULTADOS: MAPADE ACCESIBILIDAD}

Utilizando los criterios descritos en el inciso anterior se generaron varios modelos hasta encontrar el que cumplía con los criterios de decisión establecidos. Es hasta el tercer intento o modelo territorial que se cumplen los criterios de decisión. A continuación la descripción de este resultado.

\section{a. Generación de mapa de accesibilidad:}

Ya que ni el primer ni el segundo modelo cumplieron con los criterios de selección, se concluye que se debe realizar un tercer modelo. Este tercer modelo, consiste en realizar cambios en la distribución de los servicios de las rutas de transporte público únicamente, debido a que es el único factor que no ha recibido cambio, con esto tendremos un escenario en el cual se mantienen los factores de salud y educación del segundo modelo.

\footnotetext{
2 Moreno Jiménez, A. 2006. En torno a los conceptos de equidad, justicia e igualdad espacial. Huellas. La Pampa. Argentina. 11:133-142
} 
Rutas de transporte:

En este tercer modelo se realizaran cambios en las rutas de transporte, dicho cambio consistirá en la ampliación de las rutas de transporte.

\section{Centros de servicios:}

En este tercer modelo se mantienen los centros de servicios agregados en los anteriores modelos, por lo cual, en este modelo no se realiza cambio alguno.

Figura 4. Vector No.1 Rutas de transporte público.

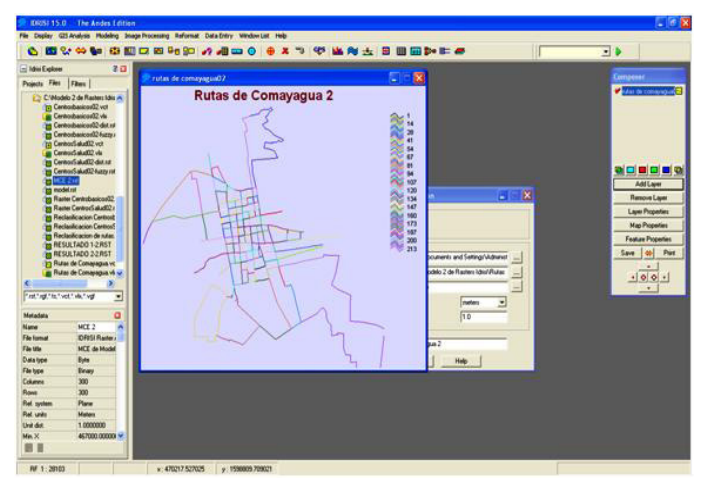

Fuente: Alcaldía Municipal de Comayagua.

\section{b. Realización del procedimiento EMC (MCE)}

Con el nuevo mapa fuzzy de rutas y usando los mapas fuzzy de salud y educación del segundo modelo, procedemos a aplicar el procedimiento del WLC, a través de la siguiente fórmula:

$$
W L C=(\text { Factor } 1 \times 0,30)+(\text { Factor } 2 \times 0,30)+(\text { Factor } 3 \times 0,40)
$$


Figura 5. Mapa No.5 - Evaluación Multicriterio Modelo No.3

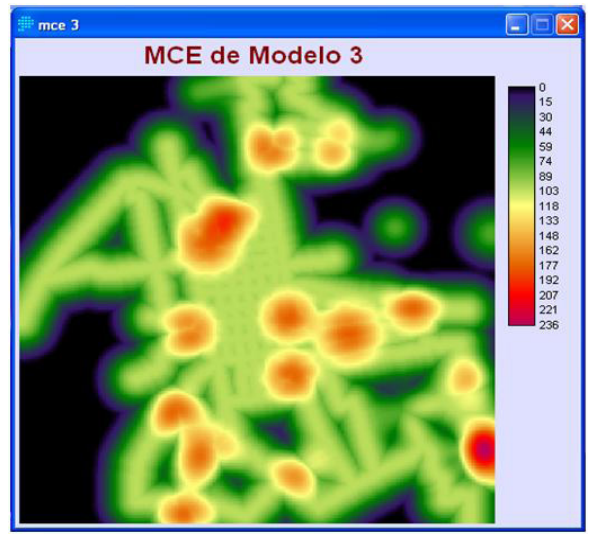

Fuente: elaboración propia.

\section{c. Generación de mapa de accesibilidad y mayor aptitud:}

Con el MCE, se recortan los resultados superponiéndolos al área de estudio (mapa catastral), con la cual, se obtiene el MAPA DE ACCESIBILIDAD, constituyendo este el resultado 3 del modelo No.3:

Figura 6. Mapa No.5 - Mapa de Accesibilidad del Modelo No.3.

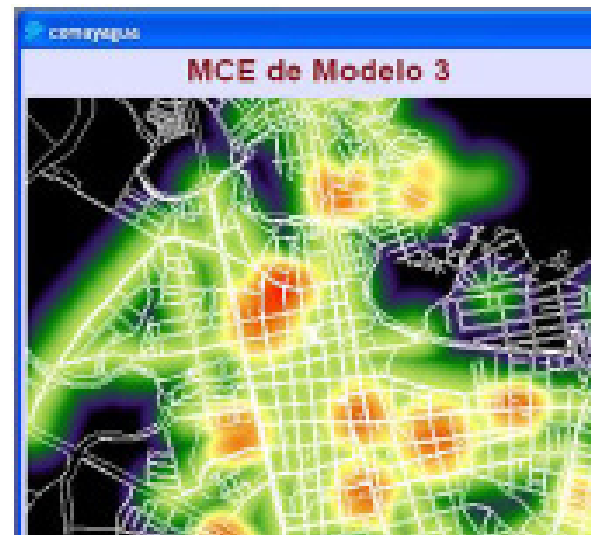

Fuente: elaboración propia. 
Priorizando el mapa de accesibilidad en las zonas de aptitud alta, usaremos el rango entre 100 y 255 como se ha realizado anteriormente. Con lo cual, obtenemos el mapa de ubicación de las zonas de mayor aptitud o accesibilidad:

Figura 7. Mapa No.6 -Mapa de las áreas con mayor aptitud o accesibilidad del Modelo No.3.

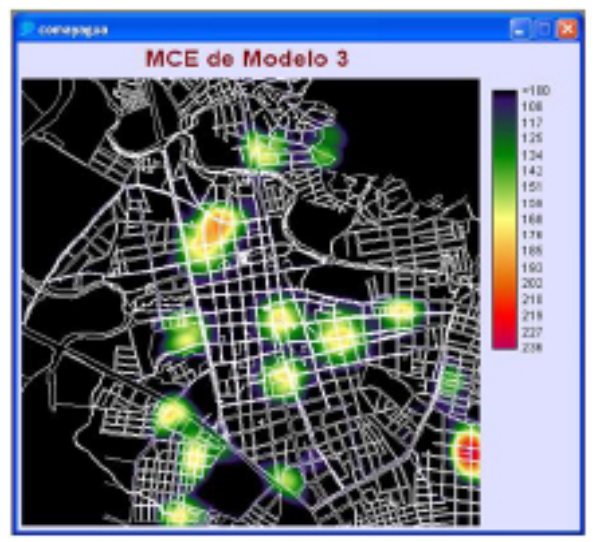

Fuente: elaboración propia.

\section{d. Reclasificación del mapa de accesibilidad y cálculo de área del mapa de acce- sibilidad :}

En este tercer modelo mantendremos los valores digitales $\mathrm{DN}=1$, para las áreas beneficiadas con los servicios en evaluación, y las áreas con valores digitales $\mathrm{DN}=0$, son las áreas sin el beneficio de estos servicios. Una vez obtenido el mapa de las áreas beneficiadas se calcula la superficie del área en kilómetros cuadrados. 
Figura 8. Imagen No.1 -Cálculo de área de beneficio

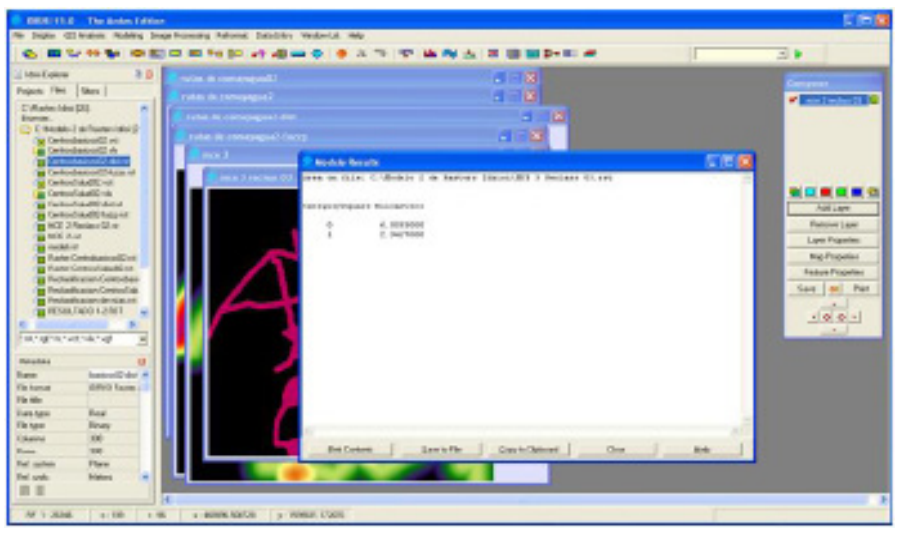

Fuente: elaboración propia.

Los cálculos de la superficie beneficiada con una accesibilidad óptima a los servicios de transporte público, salud pública y educación pública para este modelo, nos muestran los siguientes resultados:

\begin{tabular}{|l|l|l|}
\hline Area Urbana & Superficie (Km2) & Porcentaje (\%) \\
\hline Sin beneficio & 6.0583 & 67.31 \\
\hline Con beneficio & 2.9417 & 32.69 \\
\hline Total & 9.0000 & 100.00 \\
\hline
\end{tabular}

\section{ANALISIS DE RESULTADOS}

A continuación el análisis del modelo No.3, criterio a criterio:

\section{a. Mayor área de población beneficiada con servicios}

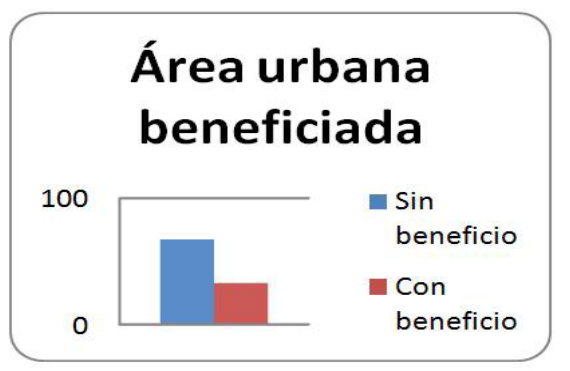

En el tercer modelo se cumplió este criterio, produciendo un aumento del área beneficiada en $32.69 \%$ (10.32\% mayor que lo resultante en el primer escenario) de la zona urbana en estudio.

Figura 9. Área urbana beneficiada. 


\section{b. Mejor distribución de servicios}

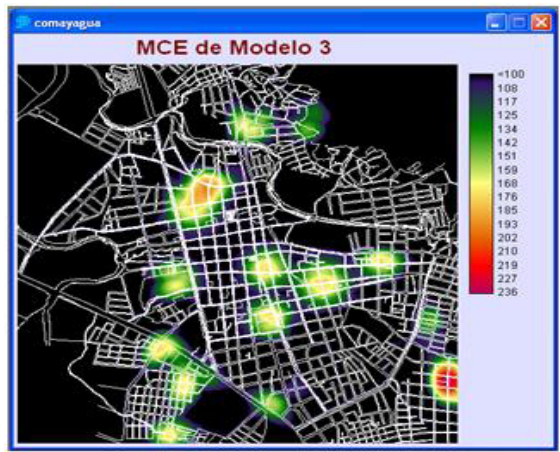

También este criterio es cumplido, ya que las zonas de mayor aptitud o accesibilidad, se encuentran más distribuidas en la zona de estudio. Lo mejor del modelo, es que los servicios se distribuyen hacia los barrios populares.

Figura 10. MCE de Modelo 3.

\section{c. Las áreas con mayor aptitud están en los barrios populares}

Finalmente, este criterio también fue cumplido, ya que la zona de mayor aptitud es un barrio popular (Colonia 21 de Abril) Después otros cuatro barrios tienen alto grado de aptitud, exceptuando el barrio abajo. 
Figura 11. Mapa No.7 - Mapa de ubicación de barrios populares del Modelo No.3

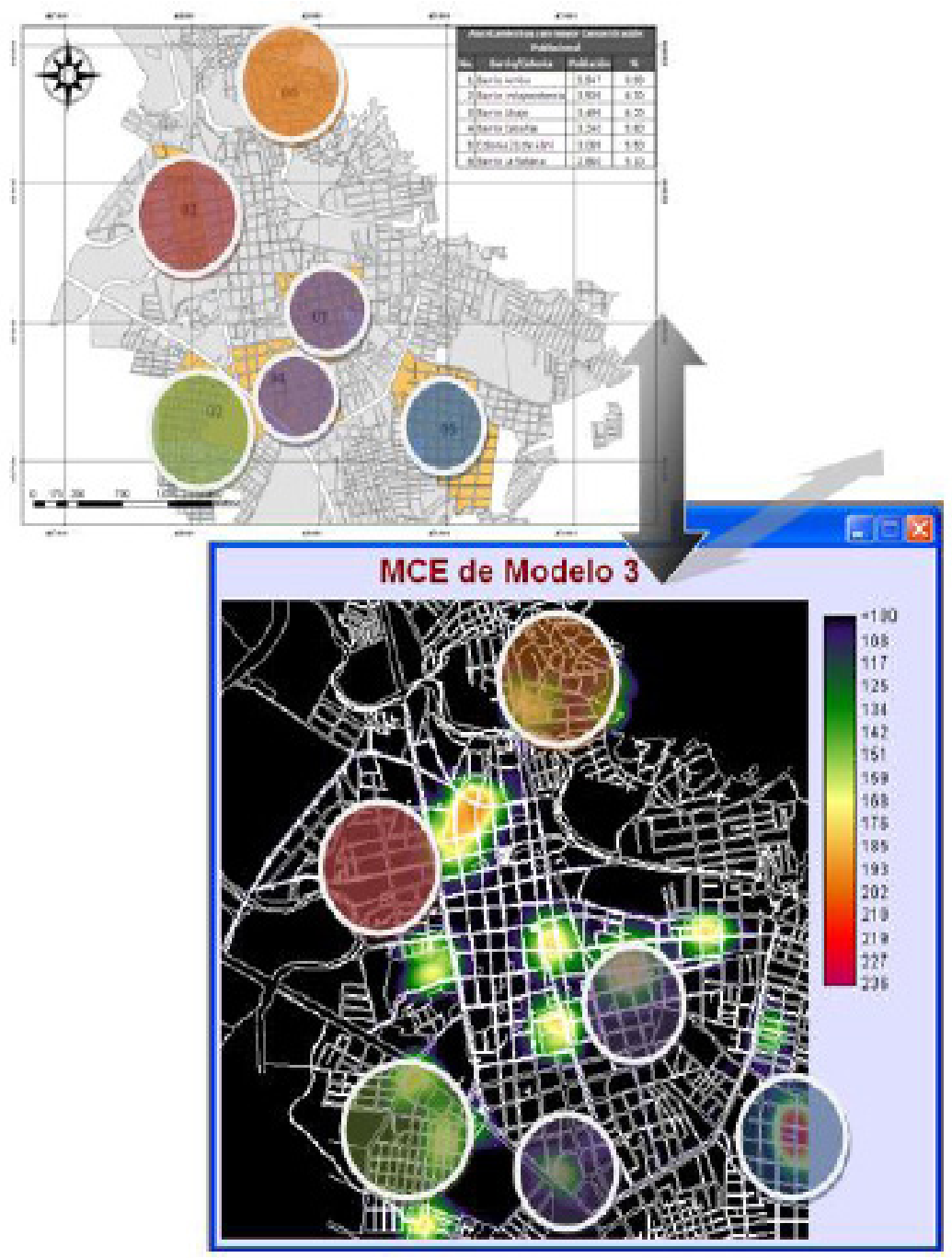

Fuente: Elaboración propia.

\section{CONCLUSIÓN SOBRE MODELO TERRITORIAL NO.3}

Se puede concluir, que los criterios se cumplen en su totalidad por este tercer Modelo Territorial, por lo que se concluye que este modelo ofrece la mayor accesibilidad a los servicios para los habitantes de barrios populares. 
Tabla 4. Cumplimiento de criterios de decisión Modelo No.3

\begin{tabular}{|l|l|l|l|}
\hline No & Criterio & $\begin{array}{l}\text { Si } \\
\text { Cumple }\end{array}$ & $\begin{array}{l}\text { No } \\
\text { cumple }\end{array}$ \\
\hline 1 & Mayor área de población beneficiada con servicios. & $\mathrm{X}$ & \\
\hline 2 & Mejor distribución de servicios & $\mathrm{X}$ & \\
\hline 3 & Las áreas con mayor aptitud están en barrios populares. & $\mathrm{X}$ & \\
\hline
\end{tabular}

Fuente: Elaboración propia.

\section{BIBLIOGRAFIA:}

- Buzai, G.D.; Baxendale, C.A. 2006. Análisis Socioespacial con Sistemas de Información Geográfica. Lugar Editorial. Buenos Aires.

- Thunen Von, Johann Heinrich Der isolierte Staat en: Beziehung auf Landwirtschaft und Nationalokönomie (Neudruck der Ausgabe 2.bzw.1.Auflage 1842 bzw, 1850). Stuttgart 1966 (4).

- Barkin, D. 1994. Reconcebir la ciudad: una nueva perspectiva de las políticas de asentamientos humanos en América Latina. En: Smolka, M.O.; Mullahy, L. (eds.) Perspectivas urbanas. Lincoln Institute of Land Policy. Cambridge.

- Bosque Sendra, J. 1992. Sistemas de Información Geográfica. Rialp. Madrid.

- Bosque Sendra, J. 2001. El uso de los sistemas de información geográfica en la planificación territorial. El Campo de las Ciencias y las Artes. Madrid. España. 138:137-174.

- Buzai, G.D. 2003. Mapas Sociales Urbanos. Lugar Editorial. Buenos Aires. 1:61-62.

- Calder, A; Greenstein, R. 2007. Política de suelo, mercados inmobiliarios y segregación espacial urbana. En: Smolka, M.O.; Mullahy, L. (eds.) Perspectivas urbanas. Lincoln Institute of Land Policy. Cambridge. pp. 323-328. 
- Eastman, J.R. 2007. La verticalización de los Sistemas de Información Geográfica. Memorias de la XI Conferencia Iberoamericana de Sistemas de Información Geográfica. Departamento de Ciencias Sociales - UNLu. Luján. pp. 183-195.

- Gómez Delgado, M.; Barredo Cano, J.I. 2006. Sistemas de Información Geográfica y evaluación multicriterio en la ordenación del territorio. Alfaomega-Rama. México.

- Moreno Jiménez, A. 2006. En torno a los conceptos de equidad, justicia e igualdad espacial. Huellas. La Pampa. Argentina. 11:133-142.

- Smolka, M. 2007. Mercados Informales de Suelo y Regularización de Asentamientos Informales, Curso Latinoamericano. Lincoln Institute of Land Policy. Lima, Perú.

- Smolka, M.O.; Mullahy, L. (eds.) 2007. Perspectivas urbanas. Lincoln Institute of Land Policy. Cambridge.

- Clarks Labs, 2004. Manual del Idrisi Kilimanjaro, Guia para SIG y procesamiento de imágenes, mayo 2004 